\title{
Correction to: Efficacy and safety of delivery of topical medication on to the frontal sinus at different head positions after frontal sinusotomy
}

\author{
Yu-Xiao Wu ${ }^{1} \cdot$ Min Wang ${ }^{1}$ (D $\cdot$ Hui Li ${ }^{1} \cdot$ Zhi-Min Xing $^{1} \cdot$ Mu-Han Shi ${ }^{1} \cdot$ Shi-En Huang ${ }^{1} \cdot$ Yan Liu ${ }^{1} \cdot$ Cong-Li Geng ${ }^{1}$
}

Published online: 6 March 2020

(c) Springer-Verlag GmbH Germany, part of Springer Nature 2020

Correction to: European Archives of Oto-Rhino-Laryngology https://doi.org/10.1007/s00405-020-05851-w

In the original publication of the article, under the experimental protocol of the section "Clinical head position study in postoperative CRS patients", the following sentence "There were 20 CRS patients, 12 male and 8 female across an age range of 19-64 years..." was published incorrectly.

The correct sentence should read as "There were 20 CRS patients, 12 males and 8 females across an age range of 19-66 years...".
Publisher's Note Springer Nature remains neutral with regard to jurisdictional claims in published maps and institutional affiliations.

The original article can be found online at https://doi.org/10.1007/ s00405-020-05851-w.

Min Wang

minwang333@sina.com

1 Department of Otorhinolaryngology Head and Neck Surgery, Peking University People's Hospital, Xi Zhi Men Nan Da Jie 11\#, Beijing 100044, People's Republic of China 